\title{
Chronic nasal congestion and hyposmia in a 22-year-old woman
}

\author{
Mark Bastianelli MD, Shaun Kilty MD
}

Cite as: CMAJ 2017 May 8;189:E667-8. doi: 10.1503/cmaj.160956

A n otherwise healthy 22-year-old woman, who is a lifelong nonsmoker, visits her family physician with a six-month history of daily bilateral nasal obstruction, hyposmia, congestion and rhinorrhea. Her symptoms began in the summer and persisted through the fall. She had no prior history of seasonal allergic symptoms.

\section{What diagnoses should be considered?}

Several diagnoses can be considered for this patient, including rhinitis (allergic or nonallergic) and chronic rhinosinusitis.

Allergic rhinitis is caused by immunoglobulin E (IgE)-mediated inflammation of nasal mucosa in response to an aeroallergen. In contrast, nonallergic rhinitis results in nasal mucosal inflammation that may be secondary to recurrent infection, medications and hormone fluctuation, as well as other triggers (e.g., tobacco smoke, and occupational or other airborne irritants). Clinically, it is distinguishable from allergic rhinitis by its pattern of symptoms, negative allergen skin prick results and the absence of other environmental sensitivities. ${ }^{1}$

According to the 2011 Canadian rhinosinusitis guideline, ${ }^{2}$ chronic rhinosinusitis occurs when two of the following major sinonasal symptoms are present: congestion, facial pain, obstruction, rhinorrhea or smell disturbance (hyposmia or anosmia) for a minimum of eight weeks, as well as objective evidence (from computed tomography $[\mathrm{CT}]$ scan or nasal endoscopy) showing signs of paranasal sinus inflammation. Chronic rhinosinusitis can be divided into two subtypes: with or without polyps. These two subtypes have different pathogenic mechanisms and may represent distinct diseases. ${ }^{2}$

The severity of patient symptoms can be assessed with the Sino-Nasal Outcome Test (SNOT). The SNOT-22 questionnaire is a 22-item self-administered measure of quality of life (www.canvasc. $\mathrm{ca} / \mathrm{pdf} / \mathrm{SNOT22}$.pdf). Its validity in showing the impact of chronic rhinosinusitis on the patient's quality of life was demonstrated in a well-designed prospective cohort study. The questionnaire can also be used to measure the outcome of surgical or medical intervention. $^{3}$

\section{What should be included on physical examination?}

According to expert panel opinion, physical examination of the nasal cavity for all patients suspected of having chronic rhinosinusitis should be performed to identify supporting signs of disease. ${ }^{2}$ An otoscope can provide a sufficient view of the nasal cavity. ${ }^{2}$ A structured assessment of the nasal cavity should be done, including evaluation of the middle meatus, nasal septum and turbinates, and the mucosal lining, noting any evidence of polyps, purulence, septal deviation, nasal secretions, mucosal changes, turbinate hypertrophy or nasal mass. ${ }^{2}$

\section{Does this patient require any investigations?}

Routine testing or imaging is not required for all patients with rhinosinusitis. However, no evidence-based guideline provides advice on when to offer imaging. In our opinion, CT should be considered in patients who have moderate or severe symptoms that last longer than eight weeks and do not respond to medical therapy or in patients who have atypical symptoms of sinusitis (e.g., proptosis and epistaxis). According to the Canadian rhinosinusitis guideline, a noncontrast CT scan of the paranasal sinuses is the preferred radiologic assessment for suspected chronic rhinosinusitis. ${ }^{2}$ Plain films of the sinuses are not sufficient, because they lack adequate sensitivity and specificity to diagnose this condition. ${ }^{2}$ In the setting of chronic rhinosinusitis, a CT will also help differentiate its subtypes and help to guide decisions about further medical treatment and the role of surgery. Based on a well-designed cost-analysis study, an upfront CT referral strategy (i.e., having the primary care provider order a CT scan before making the decision to refer) can improve the effectiveness of referrals from primary care providers to the appropriate specialist; however, this comes with an additional cost to the health care system of $\$ 1522$ per patient. ${ }^{4}$

If chronic rhinosinusitis is diagnosed, what treatments should be offered to this patient?

Intranasal corticosteroids are the mainstay of treatment for chronic rhinosinusitis because of their anti-inflammatory properties and their ability to reduce polyp size and improve nasal congestion. ${ }^{5,6}$ Daily use of topical intranasal corticosteroids often requires two to three months to achieve symptom relief. ${ }^{5}$ In a welldesigned double-blind, placebo-controlled trial, long-term topical steroids taken intranasally were shown to be safe in adults and did not result in an increase in intraocular pressure or adrenal suppression. ${ }^{5}$ In addition, according to practice guidelines and expert panel recommendation, a short course of oral steroids can be considered for patients with severe symptoms to reduce mucosal inflammation and polyp size. ${ }^{2}$ 


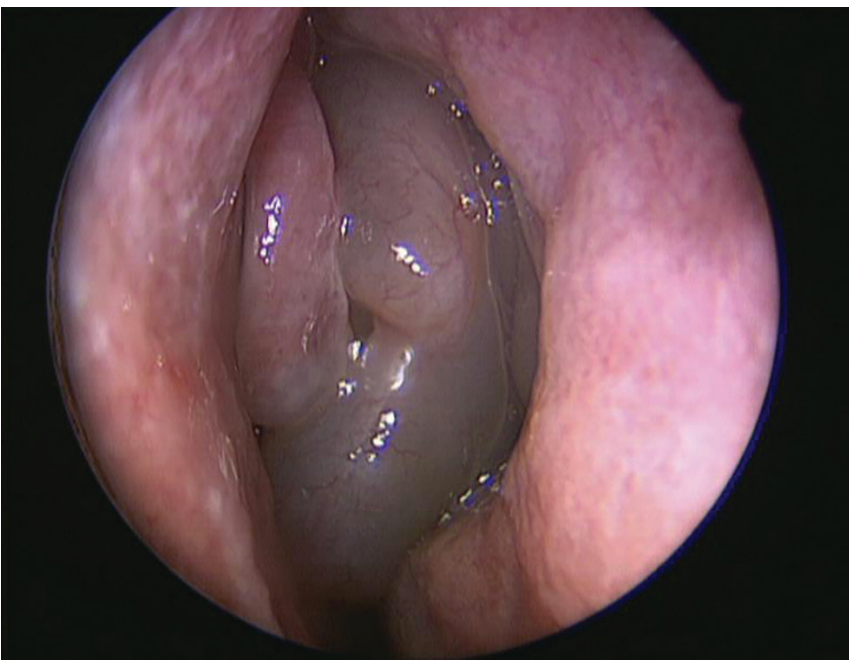

Figure 1: Nasal polyps in the left middle meatus of the nose, without purulent secretions, in a 22-year-old woman with chronic nasal congestion and hyposmia.

There is consistent evidence of the benefit of saline irrigation in patients with chronic rhinosinusitis. A Cochrane review based on eight large randomized controlled trials showed that saline is effective for symptom relief in this condition when used as the sole modality and as a treatment adjunct. ${ }^{7}$

Antibiotics should be considered in patients with substantial pain, purulence or recurrent acute-on-chronic sinusitis. ${ }^{2}$ A swab of the middle meatus of the nose may be obtained to help guide therapy, but empiric antibiotic therapy should target aerobic gram-positive and -negative bacteria as well as anaerobes. ${ }^{8}$ Amoxicillin and clavulanic acid or moxifloxacin are among the recommended first-line antibiotics. ${ }^{2}$

\section{Does this patient require referral?}

Expert opinion from the Canadian rhinosinusitis guideline suggests that the following patients with chronic rhinosinusitis should be referred to an otolaryngologist: patients whose symptoms do not respond to more than one course of medical ther- apy, those with more than three acute-on-chronic sinus infections per year, and those with severe symptoms (e.g., pain and facial swelling) or who are immunocompromised. ${ }^{2}$ These patients may require endoscopic sinus surgery to open the paranasal sinus cavities and facilitate mucociliary clearance and improve topical medication delivery.

\section{The case revisited}

The patient's score on the SNOT-22 questionnaire was 42, indicating a moderate-to-severe impact on her quality of life. Physical examination showed polypoid mucosal changes within the middle meatus (Figure 1). A CT scan of the paranasal sinuses showed polypoid mucosal thickening within the middle meatus and the ethmoid and maxillary sinuses.

Chronic rhinosinusitis with nasal polyps was diagnosed. The patient was prescribed an intranasal corticosteroid spray (two sprays per nostril daily) and advised to do saline rinses once to twice daily. At her three-month follow-up appointment, she had regained olfaction, no longer had rhinorrhea and noted that her nasal congestion was better. She continued the intranasal corticosteroid spray and saline irrigation, with a planned review in six months.

\section{References}

1. Quillen DM, Feller DB. Diagnosing rhinitis: allergic vs. nonallergic. Am Fam Physician 2006;73:1583-90.

2. Desrosiers M, Evans GA, Keith PK, et al. Canadian clinical practice guidelines for acute and chronic rhinosinusitis. J Otolaryngol Head Neck Surg 2011;40(Suppl 2): S99-193.

3. Hopkins C, Gillett S, Slack R, et al. Psychometric validity of the 22-item Sinonasal Outcome Test. Clin Otolaryngol 2009;34:447-54.

4. Kilty SJ, Leung R, Rudmik L. Economic evaluation of a computed tomography directed referral strategy for chronic rhinosinusitis. Clin Otolaryngol 2016;41:782-7.

5. Small CB, Hernandez J, Reyes A, et al. Efficacy and safety of mometasone furoate nasal spray in nasal polyposis. J Allergy Clin Immunol 2005;116: 1275-81.

6. Rotenberg BW, Zhang I, Arra I, et al. Postoperative care for Samter's triad patients undergoing endoscopic sinus surgery: a double-blinded, randomized controlled trial. Laryngoscope 2011;121:2702-5.

7. Harvey R, Hannan SA, Badia L, et al. Nasal saline irrigations for the symptoms of chronic rhinosinusitis. Cochrane Database Syst Rev 2007;(3):CD006394.

8. Brook I. Microbiology of sinusitis. Proc Am Thorac Soc 2011;8:90-100.
Competing interests: Shaun Kilty has received grants from Ondine Biomedical and Baxter Corporation, and personal fees from Baxter Corporation and Meda Pharmaceuticals. No other competing interests were declared.

This article has been peer reviewed.

The authors have obtained patient consent.
Affiliation: Department of Otolaryngology-Head and Neck Surgery, University of Ottawa, The Ottawa Hospital, Ottawa, Ont.

Contributors: Both authors contributed equally to the conception and authorship of the manuscript. Both authors approved the final version to be published and agreed to be guarantors of the work.

Correspondence to: Mark Bastianelli, mark.bastianelli@gmail.com 